\title{
Comunicación
}

\section{Osteopatía craneomandibular en un canino mestizo}

\author{
Craniomandibular osteopathy in a crosbred dog
}

\author{
Vicente Chilón-Cornejo ${ }^{1}$, Diego Diaz-Coahila ${ }^{1,2}$, Ysaac Chipayo Gonzáles $^{1}$
}

\section{Resumen}

Se presenta el caso de un paciente canino de 9 meses, de raza no definida, con disminución de la ingesta de alimento y aumento de tamaño de la parte ventral de la mandíbula. El examen radiográfico mostró proliferación ósea bilateral y en ventral del cuerpo mandibular con aumento de radiodensidad de esta zona. La lesión fue compatible con osteopatía craneomandibular, enfermedad poco común de etiología desconocida que afecta a perros jóvenes y que suele a ser autolimitante y de pronóstico favorable.

Palabras clave: radiografia, mandíbula, osteopatía, perro

\section{Abstract}

It is presented the case of a 9-month-old canine patient, of an undefined breed, with decreased feed intake and increased size of the ventral part of the mandible. Radiographic examination showed bilateral and ventral bone proliferation of the mandibular body with increased radiodensity in this area. The lesion was compatible with craniomandibular osteopathy, a rare disease of unknown etiology that affects young dogs and is usually self-limiting and has a favourable prognosis.

Key words: x-ray, mandible, osteopathy, dog

${ }^{1}$ Clínica de Animales Menores, Facultad de Medicina Veterinaria, Universidad Nacional Mayor de San Marcos, Lima, Perú

${ }^{2}$ Laboratorio de Farmacología y Toxicología Veterinaria, Facultad de Medicina Veterinaria, Universidad Nacional Mayor de San Marcos, Lima, Perú

${ }^{3}$ Email: vicente.chilon@unmsm.edu.pe

Recibido: 9 de septiembre de 2019

Aceptado para publicación: 25 de mayo de 2020

Publicado: 11 de agosto de 2020 


\section{INTRODUCCIÓN}

La osteopatía craneomandibular es una enfermedad de tipo inflamatorio, poco común, de etiología desconocida y que se caracteriza por la proliferación ósea, irregular, no neoplásica, bilateral que afecta los huesos del cráneo de perros jóvenes (Alexander, 1983; Hudson et al., 1994). Se presenta principalmente en el cuerpo y ramas mandibulares, bullas timpánicas, hueso parietal, hueso occipital y, en algunas ocasiones, en la metáfisis de huesos largos.

Los pacientes con esta patología suelen presentar dificultad para comer, dolor al abrir la boca, fiebres intermitentes, salivación excesiva. En casos avanzados se observa aumento considerable del tamaño de la mandíbula, disminución del rango de movimiento de la articulación temporomandibular, disminución de la ingesta de alimento y baja de peso (Huchkowsky, 2002; Montgomery y Simmons 2012). Ha sido reportada tanto en razas pequeñas como grandes, habiendo una mayor predisposición en el Scottish Terrier, West Highland White Terrier, Boston Terrier y Cairn Terrier (Padgett et al., 1986; Matiasovic et al., 2015).

Se presenta el caso de un canino mestizo con osteopatía craneomandibular que fue llevado a la Clínica de Animales Menores de la Universidad Nacional Mayor de San Marcos (Lima, Perú).

\section{Caso Clínico}

Se presenta en la Clínica Veterinaria de la Universidad Nacional Mayor de San Marcos un canino de raza no definida, macho, 9 meses de edad, con peso de $23 \mathrm{~kg}$, con historia de una semana de disminución del apetito y con la mandíbula hinchada.

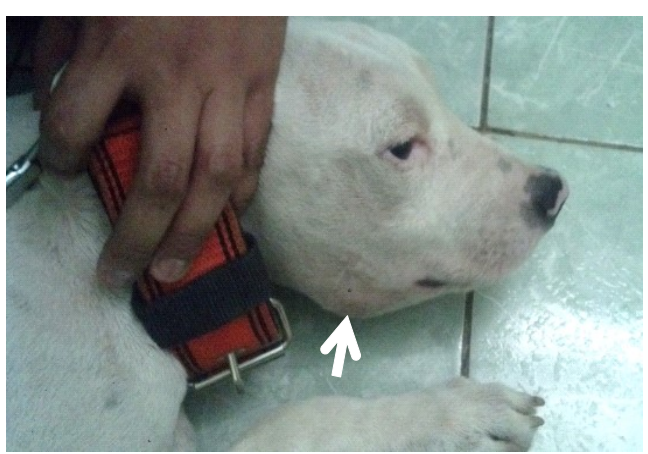

Figura 1. Canino mostrando aumento de volumen del lado derecho de la mandíbula (flecha blanca)

$\mathrm{Al}$ examen físico, el paciente presenta buena condición corporal, levemente aletargado y con un aumento de tamaño en ventral de ambos lados de la mandíbula (Figura 1). Este crecimiento se siente duro, no móvil y doloroso a la palpación. Hay dolor al intentar abrir la boca. No se detecta temperatura anormal en la zona de la mandíbula. No hay aumento de tamaño de los nódulos linfáticos. La temperatura corporal fue de $38.9^{\circ} \mathrm{C}$ y las demás constantes fisiológicas se encontraron dentro de los rangos normales.

Se realizó la evaluación hematológica sin encontrar anormalidades. La bioquímica hepática y renal solo mostró alteración en los valores de la fosfatasa alcalina $(220 \mathrm{UI} / \mathrm{L}$, valores de referencia: 0-90 UI/1). Se tomaron imágenes radiográficas de la cabeza, donde en la vista lateral derecha ligeramente oblicua se observó una reacción de periostio, proliferación ósea y aumento de radiodensidad simétrica en el borde ventral del cuerpo mandibular con pérdida de detalle de la cortical y del canal mandibular (Figura 2A). En la vista dorsoventral se observó el aumento del grosor y de radiodensidad del cuerpo mandibular. Otras estructuras óseas de la cabeza como las bullas timpánicas, articulación temporomandibular y hueso cigomático no mostraron alteraciones radiográficas (Figura 2B). 


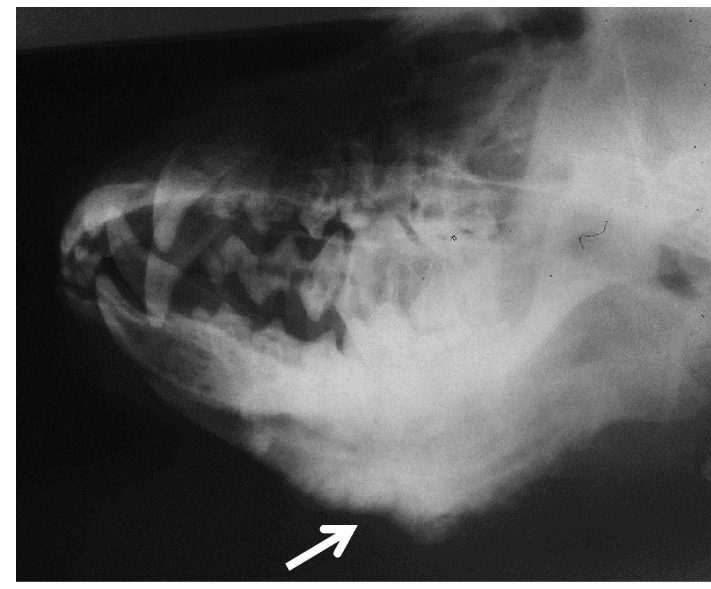

A

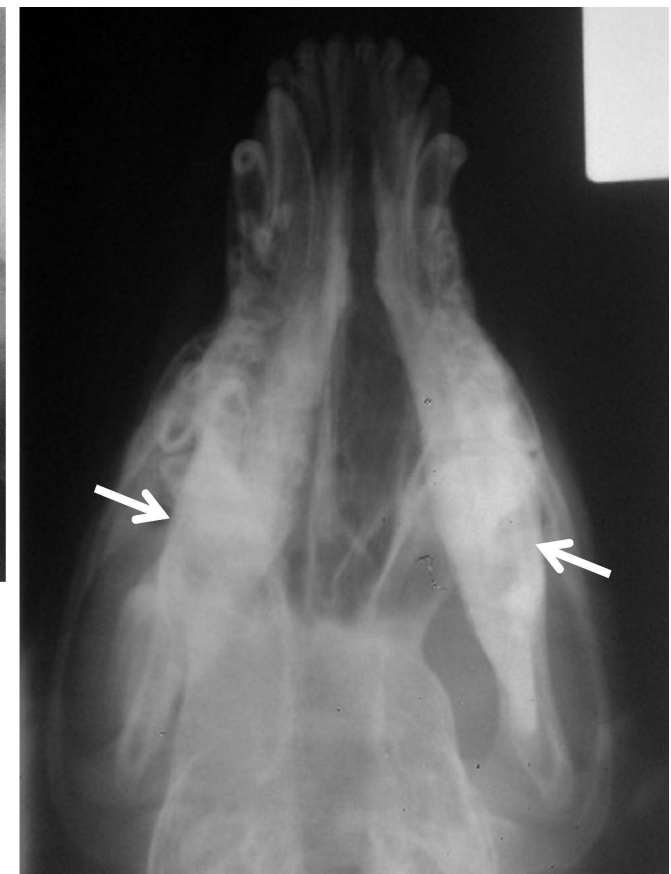

Figura 2. Imágenes radiográficas de cabeza de canino mestizo. A. Vista laterolateral derecha ligeramente oblicua mostrando proliferación ósea y aumento de radiodensidad ventral en el cuerpo mandibular (flecha blanca). B. Vista dorsoventral mostrando aumento de grosor y de radiodensidad del cuerpo mandibular (flechas blancas)

Se realizó tratamiento con corticoides usando dexametasona IM a dosis de $0.5 \mathrm{mg} / \mathrm{kg}$ de peso, seguido por 7 días de prednisona oral en dosis de $1 \mathrm{mg} / \mathrm{kg}$ cada 12 horas. Se reevaluó al día siguiente encontrándose una mejora en los signos clínicos, con disminución del dolor y aumento de la cantidad de alimento consumido. Se solicitó reevaluación a los 7 días, pero el propietario no acudió a la cita.

\section{Discusión}

La osteopatía craneomandibular, también conocida como periostitis mandibular, quijada de león, hiperostosis craneal o hiperostosis idiopática craneal suele presentarse entre los 3 a 9 meses de edad (Montgomery y Simmons, 2012). La localización más común de la lesión osteoproliferativa involucra la mandíbula, bulla timpánica y el área petrosa del temporal en el $50 \%$ de los casos y solo la mandíbula en el 33\% de los casos; mientras que en el $13 \%$ de los casos se encuentran afectadas las bullas timpánicas y el área petrosa del temporal (Franch et al., 1998; Johnson, 2010). En el presente caso, la lesión se limitó al cuerpo mandibular, con un crecimiento simétrico e irregular, sin afectar otras estructuras óseas del cráneo. El dolor generado al intentar abrir la boca para alimentarse es uno de los signos más comunes en estadios tempranos de la enfermedad (Montgomery y Simmons, 2012).

El diagnóstico de la enfermedad suele realizarse con los hallazgos clínicos más las imágenes radiográficas que muestran la proliferación ósea bilateral en las mandíbulas, bullas timpánicas, hueso temporal y occipital, 
siendo estas patognomónicas de la enfermedad (Craig et al., 2016; Beever et al., 2018). El pronóstico de la enfermedad es bueno cuando no hay una grave limitación para la alimentación que puede presentarse en caso de severo compromiso de la articulación temporomandibular (Hudson et al., 1994). La enfermedad es frecuentemente auto limitante con el cese del crecimiento de las lesiones, por lo general al año de edad, coincidiendo con el cese de la actividad fisiaria (Shorenstein et al., 2014). El tratamiento de la enfermedad comprende el uso de corticoides. En caso de complicaciones se puede adicionar el uso de fluidoterapia para corregir la deshidratación y desequilibrios electrolíticos (Montgomery y Simmons, 2012).

\section{Literatura Citada}

1. Alexander JW. 1983. Selected skeletal dysplasias: craniomandibular osteopathy, multiple cartilaginous exostoses, and hypertrophic osteodystrophy. Vet Clin North Am Small Anim Pract 13: 55-70. doi:10.1016/s0195-5616(83)50004-1

2. Beever L, Swinbourne F, Priestnall SL, Ter Haar G, Brockman DJ. 2018. Surgical management of chronic otitis secondary to craniomandibular osteopathy in three west highland white terriers. J Small Anim Pract doi: 10.1111/ jsap. 12839

3. Craig LE, Dittmer KE, Thompson KG 2016. Bones and joint disease. In: Jubb, Kennedy, and Palmer's pathology of domestic animals. $6^{\text {th }}$ ed. Elsevier. p91-92.
4. Franch J, Cesari JR, Font J. 1998. Craniomandibular osteopathy in two Pyrenean mountain dogs. Vet Rec 142: 455-459. doi:10.1136/vr.142.17.455

5. Huchkowsky SL. 2002. Craniomandibular osteopathy in a bullmastiff. Can Vet J 43: 883-885.

6. Hudson, JA, Montgomery, RD, Hathcock, JT, Jarboe, JM. 1994. Computed tomography of craniomandibular osteopathy in a dog. Vet Radiol Ultrasound 35: 94-99. doi:10.1111/j.17408261.1994.tb100194.x

7. Johnson KA. 2010. Skeletal disease. In: Ettinger SJ, Feldman EC (eds). Textbook of veterinary internal medicine disease of the dog and cat. $7^{\text {th }}$ ed. SaundersElsevier. p 831-832.

8. Matiasovic M, Caine A, Scarpante E, Cherubini GB. 2015. Imaging diagnosis-magnetic resonance imaging features of craniomandibular osteopathy in an Airedale Terrier. Vet Radiol Ultrasound 57: E27-E29. doi: 10.1111/vru.12304

9. Montgomery R, Simmons BA. 2012. Craniomandibular osteopathy. In: Bojrab MJ, Monet E (eds). Mechanisms of disease in small animal surgery. $3^{\text {rd }}$ ed. Teton NewMedia. p 2763-2779.

10. Padgett GA, Mostosky UV, Prieur DJ. 1986. Animal model: the mode of inheritance of craniomandibular osteopathy in West Highland white terrier dogs. Am J Med Genet 25: 9-13. doi:10.1002/ ajmg. 1320250103

11. Shorenstein B, Schwartz, , Kross PH. 2014. What is your diagnosis? J Am Vet Med Assoc 245: 491-492. doi:10.2460/ javma.245.5.491 